\title{
Multiplexed reverse transcription real-time polymerase chain reaction for simultaneous detection of Mayaro, Oropouche, and Oropouche-like viruses
}

\author{
Felipe Gomes Naveca ${ }^{1 /+}$, Valdinete Alves do Nascimento ${ }^{1}$, \\ Victor Costa de Souza', Bruno Tardelli Diniz Nunes², \\ Daniela Sueli Guerreiro Rodrigues², Pedro Fernando da Costa Vasconcelos ${ }^{2,3}$
}

\author{
1Fundação Oswaldo Cruz-Fiocruz, Instituto Leônidas e Maria Deane, Manaus, AM, Brasil \\ ${ }^{2}$ Ministério da Saúde, Secretaria de Vigilância em Saúde, Instituto Evandro Chagas, Ananindeua, PA, Brasil \\ ${ }^{3}$ Unversidade do Estado do Pará, Belém, PA, Brasil
}

We describe a sensitive method for simultaneous detection of Oropouche and Oropouche-like viruses carrying the Oropouche S segment, as well as the Mayaro virus, using a multiplexed one-step reverse transcription real-time polymerase chain reaction (RT-qPCR). A chimeric plasmid containing both Mayaro and Oropouche targets was designed and evaluated for the in vitro production of transcribed RNA, which could be easily used as a non-infectious external control. To track false-negative results due to PCR inhibition or equipment malfunction, the MS2 bacteriophage was also included in the multiplex assay as an internal positive control. The specificity of the multiplex assay was evaluated by Primer-Blast analysis against the entire GenBank database, and further against a panel of 17 RNA arboviruses. The results indicated an accurate and highly sensitive assay with amplification efficiency greater than $98 \%$ for both targets, and a limit of detection between two and 20 copies per reaction. We believe that the assay described here will provide a tool for Mayaro and Oropouche virus detection, especially in areas where differential diagnosis of Dengue, Zika and Chikungunya viruses should be performed.

Key words: real-time PCR - Mayaro virus - Oropouche virus - Amazon

Arthropod-borne viruses (arboviruses) are among the most important causes of emerging or re-emerging infectious diseases worldwide. Besides the great concern regarding Dengue, Zika and Chikungunya infections, epidemiological data showing the emergence of two other arboviruses known as Mayaro (MAYV) and Oropouche (OROV), suggest that these viruses are worthy of special attention, specifically in the northern region of South America (Mourão et al. 2009, Vasconcelos et al. 2009, Bastos et al. 2012, Cardoso et al. 2015). In the present study, we describe a sensitive and specific method for one-step reverse transcription real-time polymerase chain reaction (RT-qPCR) for the detection of MAYV, OROV, and other OROV-like viruses carrying the OROV S segment, coupled with an internal control.

All available and complete GenBank genome sequences for MAYV or that of the full $\mathrm{S}$ segment for OROV were used for two different nucleotide alignments, one for each target virus, using ClustalX software (Jeanmougin et al. 1998). Conserved regions were chosen and analysed for primer and probe design using Primer Express 3.0 (Applied Biosystems, Thermo Scientific, CA, USA) with the default parameters. Two prim-

doi: 10.1590/0074-02760160062

Financial support: FAPEAM PPP, DECIT/FAPEAM PPSUS, CNPq.

+ Corresponding author: felipe.naveca@fiocruz.br

Received 21 February 2016

Accepted 29 June 2016 er and probe sets were designed, according to the lower penalties score in Primer Express, targeting the NSP1 coding sequence of MAYV and the S segment of OROV. Selected primer sets were also submitted to primer-blast analysis (Ye et al. 2012) of the entire GenBank database under default parameters, except the minimum PCR product size $(50 \mathrm{bp})$. Primer pair specificity for the database parameter was set to ' $\mathrm{nr}$ ' and the organism parameter was set to 'all'. A third TaqMan MGB probe was designed as described above, targeting the coat protein (cp) gene of the Enterobacteria phage MS2 (ATCC 15597$\mathrm{B} 1$ ), used as the positive internal control (Table).

After choosing the primer and probe sets, a synthetic positive external control encompassing both MAYV and OROV target regions (Fig. 1) was ordered as a custom-made plasmid named pOROV_MAYV form IDT DNA Technology (IA, USA). The RT-qPCR target was located downstream of a T7 RNA polymerase promoter site, which allowed us to produce chimeric RNA molecules containing both targets with TranscriptAid ${ }^{\mathrm{TM}} \mathrm{T} 7$ High Yield Transcription Kit according to manufactures' instructions (Fermentas UAB, Thermo Scientific, Vilnius, Lithuania). Before the in vitro transcription of RNA, the insert and the T7 promoter site were PCR amplified (amplicon size, $925 \mathrm{bp}$ ) to limit the template size, for enhancement of in vitro RNA transcription.

The generated amplicons were precipitated with molecular biology grade polyethylene glycol $8000(20 \%$ $\mathrm{w} / \mathrm{v}$; Promega, WI, USA) for primers and dNTP removal. DNase digested in vitro transcribed RNA was quantified with a Qubit ${ }^{\circledR}$ RNA HS Assay Kit using a Qubit ${ }^{\circledR}$ 
TABLE

Oligonucleotides designed in this study

\begin{tabular}{lccc}
\hline Oligo & Sequence & Start & Stop \\
\hline MAYV_FNF & 5' CACGGACMTTTTGCCTTCA 3' & 465 & 483 \\
MAYV_FNR & 5' AGACTGCCACCTCTGCTKGAG 3' & 524 & 504 \\
MAYV_FNP & 5'(VIC) ACAGATCAGACATGCAGG 3' & 485 & 502 \\
OROV_FNF & 5' TCCGGAGGCAGCATATGTG 3' & 98 & 116 \\
OROV_FNR & 5' ACAACACCAGCATTGAGCACTT 3' & 160 & 139 \\
OROV_FNP & 5'(FAM) CATTTGAAGCTAGATACGG 3' & 118 & 136 \\
MS2_IC_FNF & 5' GCGCAGAATCGCAAATACA 3' & 1494 & 1512 \\
MS2_IC_FNR & 5' CAACAGTCTGGGTTGCCACTT 3' & 1554 & 1534 \\
MS2_IC_FNP & 5'(NED) ATCAAAGTCGAGGTGCC 3' & 1515 & 1531
\end{tabular}

Start/Stop numbers refers to the nucleotide position of Mayaro (MAYV) and Oropouche (OROV) S segment, and the Enterobacteria phage MS2 GenBank reference sequences (NC_003417.1, NC_005777.1, and NC_001417.2, respectively). All probes are TaqMan Minor Groove Binding (MGB) type with the fluorophores FAM, VIC, or NED as reporters and a non-fluorescent quencher. IDT DNA Technology synthesised all desalted primers used in this study with no further purification, whereas Applied Biosystems supplied the probes. The last digit in each oligo name was used to identify the forward (F) and reverse (R) primers, as well as the probes (P).

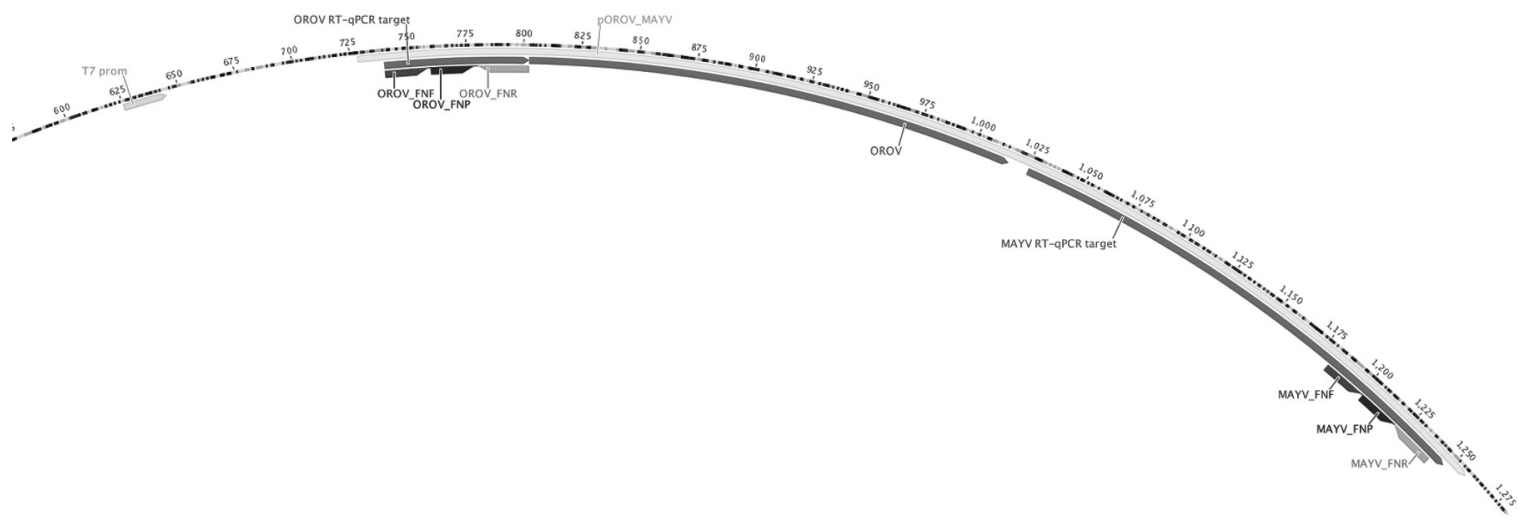

Fig. 1: chimeric plasmid pOROV_MAYV. The pOROV_MAYV plasmid contains both Mayaro (MAYV) and Oropouche (OROV) targets in the context for in vitro RNA production from the T7 promoter site. The forward and reverse primer binding sites, as well as the probe binding sites are represented by a dark grey, light grey, and a black arrow, respectively. The pOROV_MAYV plasmid was ordered form IDT DNA Technology.

2.0 Fluorometer (Invitrogen, Thermo Scientific, CA, USA). RNA quantitation and its sequence were used to calculate the exact target copy numbers with the aid of the endmemo server (endmemo.com/bio/dnacopynum. php). The in vitro transcribed RNA was used in all optimisation steps of this protocol, including primer and probe titration. We used the TaqMan ${ }^{\circledR}$ Fast Virus 1-Step master mix (Applied Biosystems) for RT-qPCR amplification with the recommended cycling parameters, $50^{\circ} \mathrm{C}$ for $5 \mathrm{~min}$ for reverse transcription, $95^{\circ} \mathrm{C}$ for $20 \mathrm{~s}$ for RT inactivation/initial denaturation, followed by 45 cycles of $95^{\circ} \mathrm{C}$ for $3 \mathrm{~s}$ and $60^{\circ} \mathrm{C}$ for $30 \mathrm{~s}$. RNA $(2 \mu \mathrm{L})$ was used as a template in a $20 \mu \mathrm{L}$ reaction, and all assays were performed using the StepOnePlus Real-Time PCR System (Applied Biosystems). The assay amplification efficiency was calculated by the standard curve method (Svec et al. 2015) using a 10-fold, 8-log, serial dilution starting at $2 \times 10^{8}$ RNA copies $/ \mu \mathrm{L}$, in duplicate.
Primer and probe specificity were evaluated against a panel of RNA arboviruses from the Brazilian National Reference Laboratory for Arbovirus at the Instituto Evandro Chagas. Viral RNA from MAYV and OROV, as well as 15 other arboviruses, from three different families were extracted from previously infected suckling mouse brain or liver with Trizol LS (Invitrogen), as recommended by the manufacturer. These included Flaviviridae (Dengue virus serotypes 1, 2, 3, and 4 and Ilheus, Rocio, Saint Louis Encephalitis, and Zika viruses), Togaviridae (Chikungunya, Trocara, and Western Equine Encephalitis viruses), and Bunyaviridae (Caraparu, Catu, Icoaraci, Jatobal, Tacaiuma, Trocara, and Utinga viruses).

After optimisation to determine the best primer and probe concentrations, we used $300 \mathrm{nM}$ of each primer and $100 \mathrm{nM}$ of each TaqMan MGB Probe. According to the standard curve results, and considering a $\mathrm{Ct}$ value lower than 38 as positive, the multiplex assay had a limit of detec- 

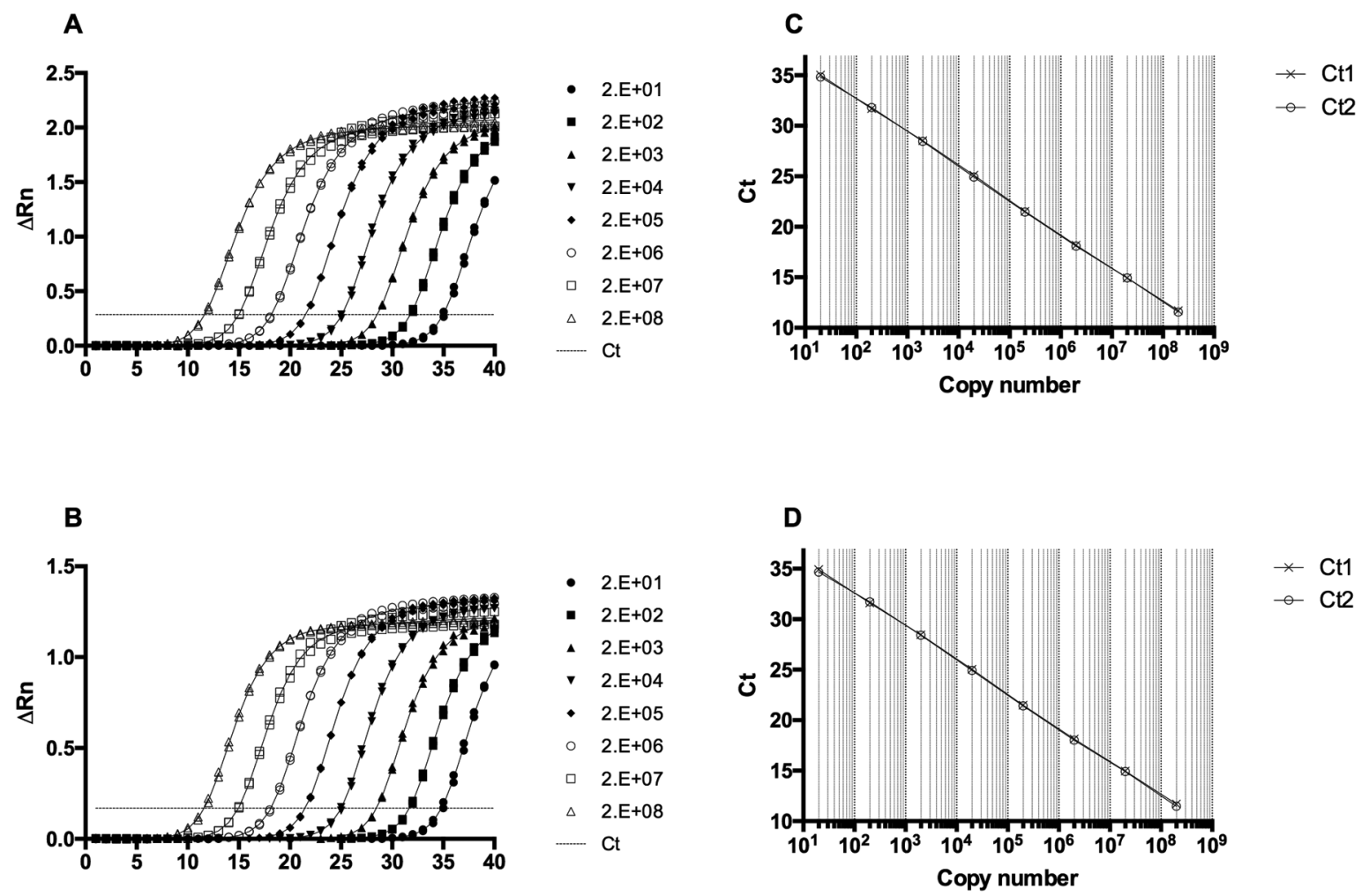

Fig. 2: reverse transcription real-time polymerase chain reaction (RT-qPCR) with serial dilutions of the chimeric in vitro transcribed RNA containing both Mayaro (MAYV) and Oropouche (OROV) targets. Amplification plots for MAYV (A) and OROV (B), and linear regression for MAYV (C) and OROV (D) for ten-fold, 8-log, dilutions from 20 to 2E+08 copies, in duplicate. PCR efficiency in the multiplex assay was calculated with StepOnePlus Software v2.2 to be $98.642 \%$ [slope: -3.355 , R2: 1] for MAYV, and 99.181\% [slope: $-3.341, \mathrm{R} 2: 1$ ] for OROV. Ct $=$ cycle threshold. Fluorescence values were exported to MS Excel and plotted using GraphPad Prism 6.0.

tion between 2-20 copies (Mean Ct values, 34.9-38.3 and 34.8-38.1 for MAYV and OROV, respectively). No considerable loss in sensitivity was observed in the multiplex format in comparison to single reactions. In fact, both MAYV and OROV amplification efficiencies showed excellent results and reproducibility in the multiplex format (Fig. 2).

As expected, no amplification was observed when the supernatant of uninfected cell lines, commonly used for arboviral isolation (C6/36 and Vero), or other arboviral RNA (those previously specified) were used as templates for the RT-qPCR reaction, with the exception of the Jatobal virus, an OROV reassortant virus, that was amplified with the OROV set. In those negative samples, only the MS2 amplification curve was consistently observed.

Bunyaviruses such as OROV possess a segmented genome encompassing three RNA segments referred to as L (large), M (medium), and S (small). This characteristic, also found in influenza and reoviruses, results in evolution through segment reassortment, which might culminate in the generation of a new virus (Briese et al. 2013). This phenomenon was observed in at least four bunyaviruses that have the S segment of OROV. The reassortant OROV-like virus Jatobal that has an $\mathrm{S}$ segment with more than $95 \%$ similarity to that of the OROV prototype (Saeed et al. 2001). In addition Madre de Dios virus (Ladner et al. 2014), Iquitos virus (Aguilar et al. 2011), and Perdoes virus (Tilston-Lunel et al. 2015) share $\mathrm{S}$ and $\mathrm{L}$ segments with OROV. These OROV-like viruses could also be amplified by the primer and probe sets designed in this study. Primer-Blast analysis with the OROV primer set returned 104 OROV sequences having a perfect match, and another 10 sequences of Jatobal, Iquitos, Madre de Dios, and Perdoes virus without a mismatch. Another 36 OROV sequences showed one or two mismatches related to the primer sequences. Regarding the MAYV primer set, primer-Blast analysis returned 37 sequences with a perfect match and 12 with one mismatch (Supplementary data). For the OROV set, as well as for the MAYV set, non-specific matches were not observed.

MAYV is a member of the Alphavirus genus in the Togaviridae family; it is closely related to the Chikungunya virus, and since its initial description, MAYV has been associated with cases of self-limited illness, presenting fever, rash, and severe arthralgia as the most common symptoms (Azevedo et al. 2009, Abad-Franch et al. 2012, Mourão et al. 2012). OROV belongs to the Orthobunyavirus genus, in the Bunyaviridae family, and is often associated with outbreaks of febrile acute Dengue-like disease. For several years, Oropouche fever was considered the second most common arboviral disease in Brazil, surpassed only by dengue fever (Figueiredo 2007, Vasconcelos et al. 2011). Nevertheless, the current 
Brazilian epidemiological scenario indicates that Zika virus infections have assumed this position. Therefore, in the context of different arboviruses cocirculating, the appropriate diagnosis of acute febrile illness, based only on clinical examination, becomes even more difficult.

Nucleic acid amplification tests (NAATs) have revamped diagnostics of infectious diseases, not only through its inherent superior sensitivity and specificity (when properly optimised), but also because of its feasibility in epidemic situations. Moreover, critical information required for their development (i.e., pathogen nucleotide sequences) is usually unrestrictedly available in public databases. Thus, the necessity for fast, sensitive, and reliable molecular tests for emerging arboviruses led us to develop the protocol described in this study.

In this study, we evaluated the use of a multiplexed RT-qPCR protocol with positive and negative viral controls. Viral RNA was obtained from cell supernatants, mouse brain, or liver, in addition to in vitro transcribed RNA, but no human clinical samples were analysed. Detection of viral RNA direct from clinical specimens is usually more challenging due to lower viral loads in comparison to what is found in the supernants of infected cells and in the tissues of inoculated mice, however, the sensitivity observed for this optimised protocol $(<20$ copies per reaction) seems to be sufficient for this application. In fact, this protocol is already being used in another study to detect human Oropouche cases in the state of Amazonas (R Figueiredo, personal communication).

In conclusion, we have developed an accurate, reproducible, and highly sensitive one-step RT-qPCR protocol for the detection of two emerging arboviruses. We believe this protocol, together with other NAATs already published, will help reduce the number of misdiagnoses of acute febrile cases that are under the dengue umbrella.

\section{ACKNOWLEDGEMENTS}

To the Program for Technological Development in Tools for Health (PDTIS FIOCRUZ), for use of its facilities, and specifically the ILMD real-time PCR platform. All oligonucleotides designed in this study were applied for a patent of invention at the National Institute of Industrial Property of Brazil - INPI (www.inpi.gov.br), under number BR1020170071456.

\section{AUTHORS' CONTRIBUTION}

FGN and VAN - Conceived, designed and optimised the assay; VCS - conducted the LOD experiments; FGN, BTDN and DSGR - responsible for the specificity tests; FGN and PFCV - wrote the first and the final version of the paper. All authors were involved in the interpretation of the data and participated in the writing of the manuscript.

\section{REFERENCES}

Abad-Franch F, Grimmer GH, de Paula VS, Figueiredo LTM, Braga WSM, Luz SLB. Mayaro virus infection in Amazonia: a multimodel inference approach to risk factor assessment. PLoS Negl Trop Dis. 2012; 6(10): e1846.
Aguilar PV, Barrett AD, Saeed MF, Watts DM, Russell K, Guevara C, et al. Iquitos virus: a novel reassortant Orthobunyavirus associated with human illness in Peru. PLoS Negl Trop Dis. 2011; 5(9): e1315.

Azevedo RSS, Silva EVP, Carvalho VL, Rodrigues SG, Nunes-Neto JP, Monteiro H, et al. Mayaro fever virus, Brazilian Amazon. Emerg Infect Dis. 2009; 15(11): 1830-2.

Bastos MS, Figueiredo LTM, Naveca FG, Monte RL, Lessa N, Figueiredo RM, et al. Identification of Oropouche Orthobunyavirus in the cerebrospinal fluid of three patients in the Amazonas, Brazil. Am J Trop Med Hyg. 2012; 86(4): 732-5.

Briese T, Calisher CH, Higgs S. Viruses of the family Bunyaviridae: are all available isolates reassortants? Virology. 2013; 446(1-2): 207-16.

Cardoso BF, Serra OP, Heinen LBS, Zuchi N, de Souza VC, Naveca FG, et al. Detection of Oropouche virus segment $S$ in patients and in Culex quinquefasciatus in the state of Mato Grosso, Brazil. Mem Inst Oswaldo Cruz. 2015; 110(6): 745-54.

Figueiredo LTM. Emergent arboviruses in Brazil. Rev Soc Bras Med Trop. 2007; 40(2): 224-9.

Jeanmougin F, Thompson JD, Gouy M, Higgins DG, Gibson TJ. Multiple sequence alignment with Clustal X. Trends Biochem Sci. 1998; 23(10): 403-5.

Ladner JT, Savji N, Lofts L, Travassos da Rosa A, Wiley MR, Gestole $\mathrm{MC}$, et al. Genomic and phylogenetic characterization of viruses included in the Manzanilla and Oropouche species complexes of the genus Orthobunyavirus, family Bunyaviridae. J Gen Virol. 2014; 95(5): 1055-66.

Mourão MPG, Bastos MS, Figueiredo RM, Gimaque JBL, Galusso EDS, Kramer VM, et al. Mayaro fever in the city of Manaus, Brazil, 2007-2008. Vector Borne Zoonotic Dis. 2012; 12(1): 42-6.

Mourão MPG, Bastos MS, Gimaque JBL, Mota BR, Souza GS, Grimmer GHN, et al. Oropouche fever outbreak, Manaus, Brazil, 2007-2008. Emerg Infect Dis. 2009; 15(12): 2063-4.

Saeed MF, Wang H, Suderman M, Beasley DW, Travassos da Rosa $\mathrm{A}, \mathrm{Li} \mathrm{L}$, et al. Jatobal virus is a reassortant containing the small RNA of Oropouche virus. Virus Res. 2001; 77(1): 25-30.

Svec D, Tichopad A, Novosadova V, Pfaffl MW, Kubista M. How good is a PCR efficiency estimate: recommendations for precise and robust qPCR efficiency assessments. Biomol Detect Quantif. 2015; 3: 9-16.

Tilston-Lunel NL, Hughes J, Acrani GO, da Silva DEA, Azevedo RSS, Rodrigues SG, et al. Genetic analysis of members of the species Oropouche virus and identification of a novel M segment sequence. J Gen Virol. 2015; 96(7): 1636-50.

Vasconcelos HB, Azevedo RSS, Casseb SM, Nunes-Neto JP, Chiang JO, Cantuária PC, et al. Oropouche fever epidemic in northern Brazil: epidemiology and molecular characterization of isolates. J Clin Virol. 2009; 44(2): 129-33.

Vasconcelos HB, Nunes MRT, Casseb LMN, Carvalho VL, da Silva EVP, Silva M, et al. Molecular epidemiology of Oropouche virus, Brazil. Emerg Infect Dis. 2011; 17(5): 800-6.

Ye J, Coulouris G, Zaretskaya I, Cutcutache I, Rozen S, Madden TL. Primer-BLAST: a tool to design target-specific primers for polymerase chain reaction. BMC Bioinformatics. 2012; 13: 134. 